Submission ID: 43764

\title{
Modern Mathematical Support of the Transient Electromagnetic Soundings
}

V.S. Mogilatov* (Trofimuk Inst. of Petroleum Geology \& Geophysics), A. Zlobinskiy (STC ZaVeTGEO)

\section{SUMMARY}

It is necessary to support mathematically any novations in the electromagnetic methods by the transient field, which appear due to peculiar methodological considerations either under process conditions, or just under terrain conditions (complex wilderness, sea, densely populated cultivated area). Electrical exploration has a steady use in the sea, and we offer new mathematical support. The operational three-dimensional approach is a large problem in the transient geoelectromagnetic soundings. We are developing the new solutions using the Born approximation and the tomographic approach. 


\section{Современное математическое обеспечение зондирований становлением (3С)}

В.С. Могилатов* (Институт нефтегазовой геологии и геофизики СО РАН. Новосибирский государственный университет), А.В. Злобинский (ООО «Научно-техническая компания ЗаВеТ-ГЕО»)

\section{Введение}

Для более широкого использования электроразведки в геофизической практике необходимо своевременно поддержать с математической стороны любые новации в электроразведке 3С, возникающие по оригинальным методологическим соображениям либо по технологическим условиям, либо просто по условиям на местности (сложная дикая местность, море, густонаселенная окультуренная зона). При этом, электроразведчики должны иметь возможность свободно видоизменять конфигурации приемно-питающих установок.

Устойчиво применяется морская электроразведка методом ЗС. Там есть методики с горизонтальными, вертикальными и наклонными электрическими линиями при расположении элементов установки на дне, на поверхности, на льду и в толще морской воды. Универсальное математическое обеспечение для подобных конфигураций обеспечило бы и нужды наземноскважинной электроразведки в режиме становления.

Конечно, давно уже назрела необходимость не одномерного подхода при интерпретации данных ЗС. Эта проблема решается не просто. Прямая трехмерная задача для зондирований становлением является все еще мало доступной для широкого круга геоэлектриков. Это высокотехнологичный математический продукт, требующий весьма квалифицированного обслуживания, обычно, даже авторского сопровождения и больших вычислительных ресурсов. Особенно заметно отсутствие общепризнанного подхода к решению трехмерной обратной задачи. Очевидный путь, который состоит в использовании процедуры решения прямой трехмерной задачи для подбора определенным образом параметризованной трехмерной неоднородности, мало устраивает электроразведчиков из-за медлительности такого процесса.

Мы предлагаем подход, который состоит в прямой инверсии (без явного решения прямой задачи) большого количества данных на основе приближенного линеаризованного представления прямой задачи. Такая процедура легко укладывается в рамки томографической инверсии. Нельзя сказать, что такой подход нов. Мы сами предлагали это довольно давно, еще в 2000 году [Могилатов и Эпов, 2000], но также за последние годы регулярно появляются работы в этом направлении (например, [Жданов, 2007], [Персова и др., 2010]). В рамках такого подхода (в борновском приближении) нетрудно получить весьма оперативные процедуры решении прямой трехмерной задачи, которые, если угодно, можно использовать и для быстрого подбора трехмерной неоднородности. Линеаризованный, томографический подход обещает создание крайне оперативного, общедоступного и легко тиражируемого математического аппарата трехмерной инверсии, что поможет сделать 3D-электроразведку контролируемым стандартным производственным методом.

\section{Универсальное матобеспечение одномерного характера для ЗС}

Итак, программа Выбор-ЗС обеспечивает прямое моделирование процесса установления в многослойной (одномерной) поляризующейся и анизотропной Земле от произвольного, задаваемого токовыми отрезками источника. Токовые отрезки могут образовывать связанные или несвязанные, замкнутые или заземленные последовательности. Источник возбуждается синхронно импульсом произвольной формы. Источник может быть помещен на любой другой границе, помимо дневной (очевидное применение - морские работы). Производится расчет всех компонент электромагнитного поля. Причем, горизонтальные компоненты рассчитываются по указанному направлению. В зависимости от датчика (приемная электрическая линия, индукционный датчик, магнитометр) рассчитанный сигнал умножается 
на соответствующий, вводимый в программу, коэффициент (длина линии, эффективная площадь датчика, коэффициент магнитометра) для трансформации сигнала в ЭДС. Приемник также может быть помещен на любую границу (фактическую или фиктивную) геоэлектрической среды, в частности, в воздух на некоторую высоту. Процесс установления может быть рассчитан с учетом анизотропии проводимости и процесса вызванной поляризации (ВП) по модели Cole-Cole. Выбор-3С - универсальное средство прямого моделирования 3С, которое всегда выручит «на первый случай», до создания специализированного матобеспечения, если применена новая экспериментальная схема. Поясним использование программы на примерах (рис. 1).
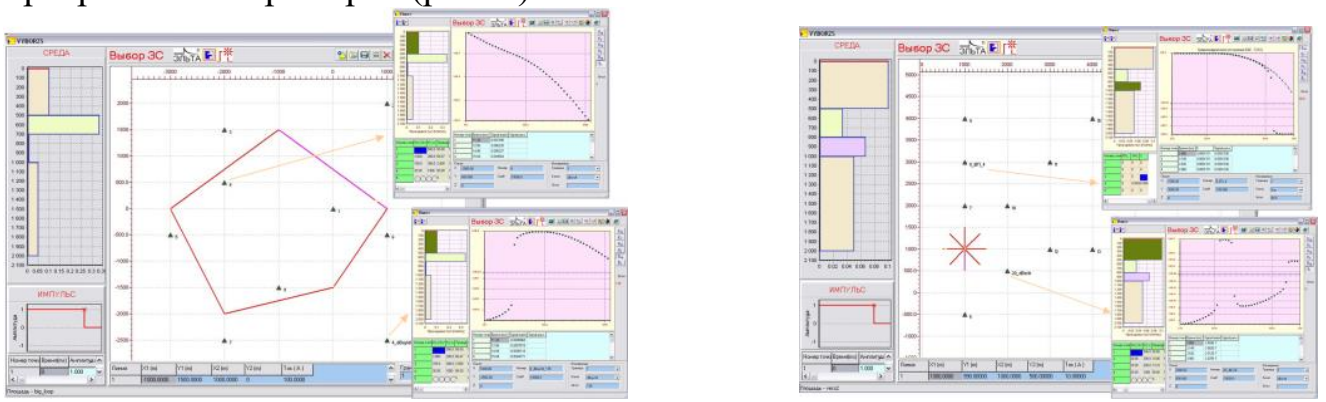

Рисунок 1 Большая петля и круговой электрический диполь (КЭД) в программе Выбор-ЗС

Представленная выше программа Выбор-ЗС предоставляет, конечно, широкие возможности для новаций в электромагнитных импульсных зондированиях. Однако, источник, даже сложный, предусматривается «плоским» и должен размещаться в одной горизонтальной плоскости, хотя бы и погруженной. Таким образом, в программе Выбор-ЗС не рассматривается важный случай источников - вертикальные электрические линии, которые используются на практике в море и (в меньшей степени) для наземно-скважинных исследований. Особое значение такой программы состоит в том, что появляется возможность оценить влияние отклонений в реальной приемно-питающей установке ABMN от горизонтали или вертикали.
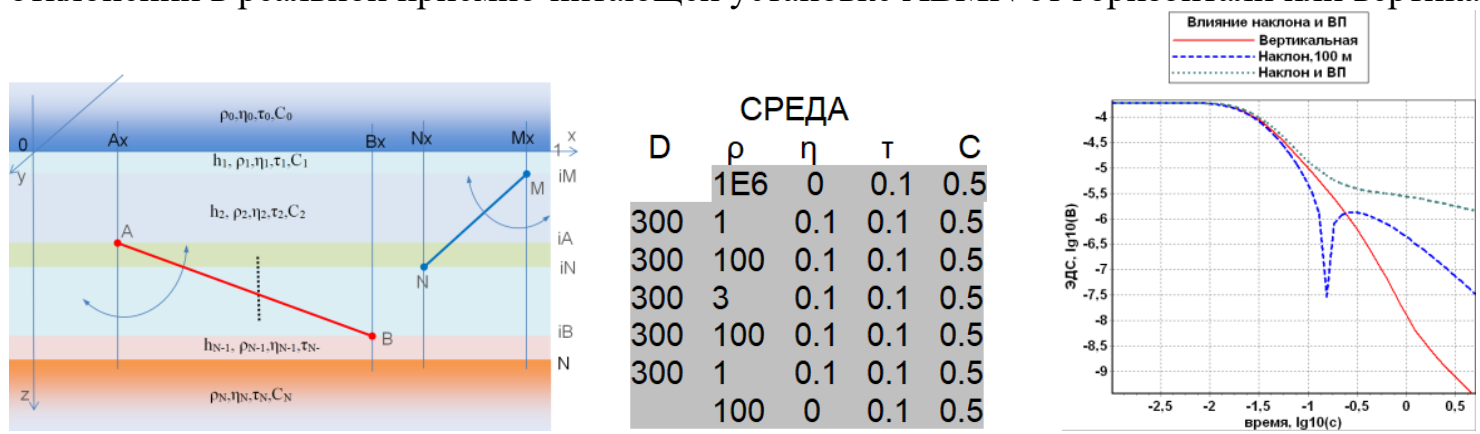

Рисунок 2 Общая модель и пример среды и расчета становления

На рис. 2 показано размещение питающей и приемной линий в горизонтально-слоистой среде, а также приведен конкретный расчет для наземно-скважинной электроразведки. Источник помещен в скважину, пересекающую пачку поляризующихся слоев, а приемная линия располагается на дневной поверхности. Сравниваются устанавливающиеся сигналы для вертикальной линии, с отклонением от вертикали и с учетом ВП. Как видим, сигналы радикально меняются.

\section{Оперативное трехмерное матобеспечение в борновском приближении}

Один из наиболее используемых подходов численного моделирования электромагнитных полей основывается на линеаризации. При этом ограничиваются нулевым и первым разложения исходного интегрального уравнения. Этот подход обычно называют приближением Борна. Рассмотрим кратко эту процедуру. Предположим, что в некотором объеме действует некий возмущающий по отношению к базовой (референтной) модели фактор, зависящий от электрического поля линейно, например, возмущение проводимости. В 
этой области первое уравнение Максвелла после выключения источника будет выглядеть следующим образом:

$$
\operatorname{rot} \mathbf{H}=\sigma_{0}(z) \cdot \mathbf{E}+\Delta \sigma(x, y, z) \cdot \mathbf{E} .
$$

Если в дополнительном члене заменить полное поле нормальным, одномерным, то мы теперь имеем одномерную задачу с распределенным сторонним током. Такую задачу мы умеем решать и приводить ее решение к быстрой численной реализации [Могилатов, 2014].

Подобный алгоритм реализован, и процедура внедрена в известную систему обработки данных зондирований в ближней зоне (ЗСБ) Подбор. На рис. 3 показаны соответствующие элементы интерфейса системы Подбор 5.
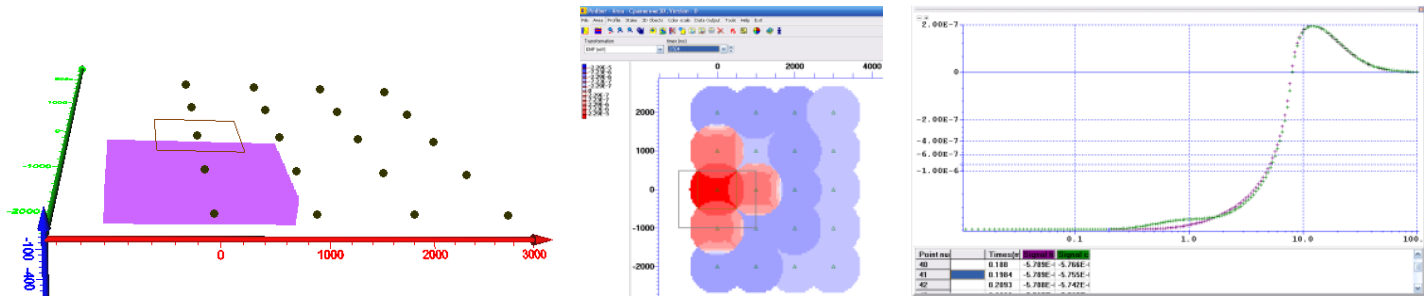

Рисунок 3 Оперативная процедура решения трехмерной прямой задачи в составе системь Подбор 5

\section{Томографическая обработка данных 3С}

Располагая линейной прямой задачей, мы можем составить линейную систему, связывающую полевые данные и неизвестные возмущения проводимости в некотором количестве пространственных элементов обследуемой области среды. Давно разработаны методы обращения больших недоопределенных или переопределенных систем (например, [Уилкинсон и Райнш, 1976]). Добавив к этому характерные приемы описания среды и способы представления результатов инверсии, мы можем претендовать, в целом, на томографический подход к решению обратной задачи.

Рассмотрим пример. Во вмещающей среде (слой 1000 м, УЭС 100 Ом*м на основании с УЭС 1000 Ом*м) неоднородность занимает по вертикали диапазон от 300м до 600м, УЭС 30 Ом*м. На рис. 4 показана неоднородность в плане и система наблюдений от двух генераторных петель (а). Синтетические данные для этой модели были рассчитаны методом конечных элементов М.Г.Персовой. На первом этапе мы полагаем, что положение неоднородности (и даже ее наличие) нам не известны. В таком случае мы подозреваем широкую область в районе зондирований - 6000х6000 м. Для начала «раскидываем» грубую томографическую сеть, 6х6. Результат представлен на рис. 4(б), и положение неоднородности определено. Теперь мы можем рассмотреть гораздо меньшую область с подробным шагом (в).

a)

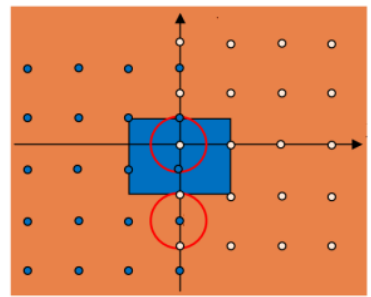

б)

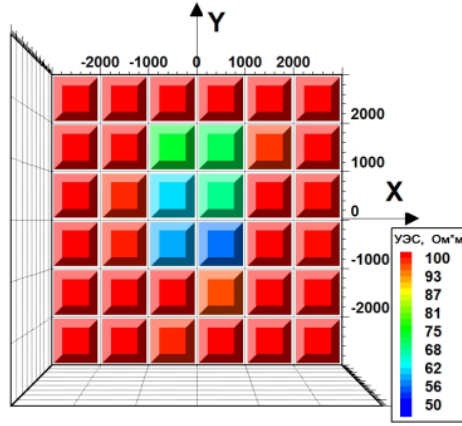

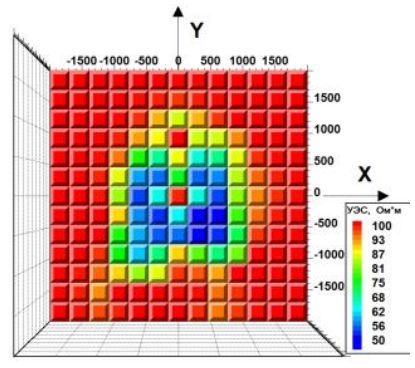

B)

Рисунок 4 Модель и схема регистращии сигнала (а); результат грубой инверсии (б); томографическая инверсия с подробной сеткой (в) 
Мы можем показать еще довольно эффектный результат, когда по площадным регистрациям переходного сигнала от закрепленных источников восстанавливается контур сложной неоднородности, такой как на рис. 5. Вмещающая среда такая же, как в предыдущем примере. Использовались пять генераторных петель, отклик регистрировался в 36 пунктах сети наблюдений с шагом 400 м (рис.5).
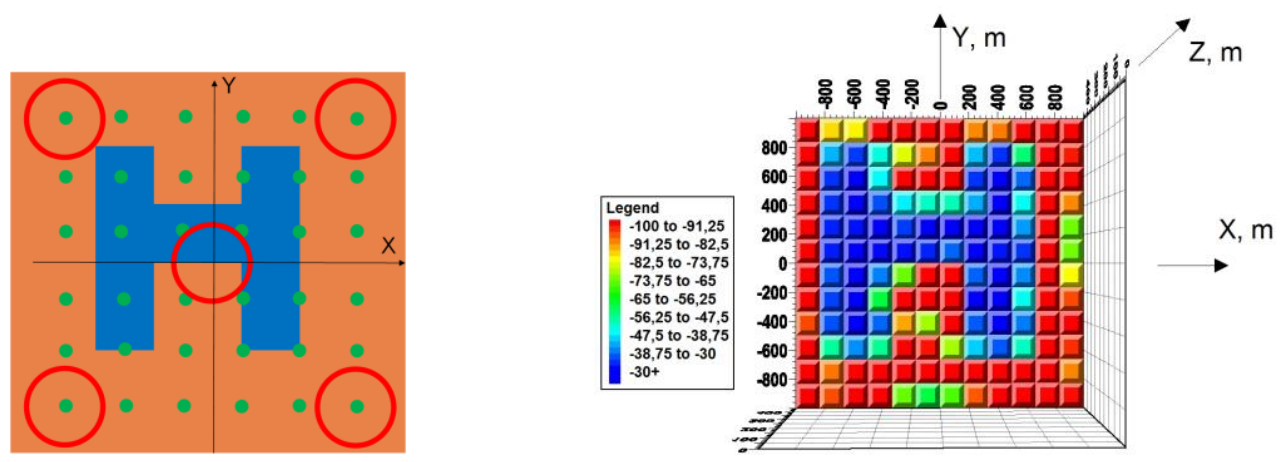

Рисунок 5 Сложная неоднородность. Инверсия переходных сигналов от иентральной генераторной петли только

Нужно отметить слабость этого численного эксперимента. Синтетические данные мы получили, воспользовавшись линеаризованным представлением прямой задачи (прямая задача в борновском приближении). Однако мы внесли помехи в виде несовпадения линий томографической сетки $(12 \mathrm{x} 12)$ с границами неоднородности. Так или иначе, мы получили и некоторый методический результат. Оказалось, что инверсия с пятью генераторными петлями (впрочем, в этом случае использовались только 9 точек наблюдения - всего 45 кривых) не улучшает инверсию по сравнению с данными от одного, центрального источника. Дело в том, что относительный аномальный эффект растет с увеличением разноса, а данные, полученные вблизи генераторных петель, только мешают.

\section{Выводы}

Математические основания зондирований становлением электромагнитного поля считались долгое время нетривиальными, и программное обеспечение ЗС всегда на шаг-другой отставало от потребностей практики. Особенно это касается трехмерного подхода. Если ситуация с прямым моделированием исправляется, то оперативная трехмерная интерпретация не имеет общепринятого подхода. Мы тут показали возможность томографического подхода, на наш взгляд, вполне работоспособного и способного реализоваться в тиражируемом и легко распространяемом математическом обеспечении.

\section{Библиография}

Жданов М.С. [2007] Теория обратных задач и регуляризации в геофизике. Научный мир, Москва.

Могилатов В. С. [2014] Импульсная геоэлектрика. Изд. НГУ, Новосибирск.

Могилатов В.С. и Эпов М.И. [2000] Томографический подход к интерпретации данных геоэлектромагнитных зондирований. Физика Земли, № 1, 78-86.

Персова М.Г., Соловейчик Ю.Г., Домников П.А. и Симон Е.И. [2010] О томографическом подходе к интерпретации данных электромагнитных зондирований в трехмерных средах. В Материалах $\mathrm{X}$ международной конференции «Актуальные проблемы электронного приборостроения» ФПЭП-2010. Изд-во НГТУ, Новосибирск, Т.6, 150-155.

Уилкинсон Дж. и Райнш К. [1976] Справочник алгоритмов на языке Алгол. Пер. с анг. Машиностроение, Москва. 


\section{EAGE}

\section{References}

Zhdanov Michael S. [2002] Geophysical inverse theory and regularization problem. Amsterdam: Elsevier. 710 p.

Mogilatov V.S. [2014] Pulse geoelectrics. Novosibirsk, NSU. 182 p.

Mogilatov V.S., Epov M.I. [2000] Tomograficheskij podhod k interpretacii dannyh geoehlektromagnitnyh zondirovanij. Fizika Zemli, № 1, 78-86.

Persova M.G., Solovejchik YU.G., Domnikov P.A. i Simon E.I. [2010] O tomograficheskom podhode k interpretacii dannyh ehlektromagnitnyh zondirovanij $\mathrm{v}$ trekhmernyh sredah. V Materialah $\mathrm{H}$ mezhdunarodnoj konferencii «Aktual'nye problemy ehlektronnogo priborostroeniya» FPEHP-2010. Izd-vo NGTU, Novosibirsk, T.6, 150-155.

Wilkinson J.H., Reinsch C. [1971] Handbook for Automatic Computation. Volume II: Linear Algebra. Springer. 448 p. 